Doi 2262

\title{
CORRIGENDA
}

\section{Discovery and development of sorafenib: a multikinase inhibitor for treating cancer}

Scott Wilhelm, Christopher Carter, Mark Lynch, Timothy Lowinger, Jacques Dumas, Roger A. Smith, Brian Schwartz, Ronit Simantov and Susan Kelley

Nature Reviews Drug Discovery 5, 835-844 (2006); doi:10.1038/nrd2130

On page 836, the following sentence implies that an assay reported in 1999 by McDonald and colleagues was used to discover Raf kinase inhibitors that led to sorafenib: "When the project team was formed in 1994, the reagents and assays were available to identify a Raf kinase inhibitor. A scintillation proximity assay for the high-throughput screening (HTS)

and identification of selective Raf/MEK/ERK enzyme inhibitors had already been developed by McDonald et al. at

Glaxo-Wellcome Inc., showing that this approach was feasible ${ }^{32 . "}$

The authors wish to correct the text as follows: "When the project team was formed in 1994, the reagents and scintillation proximity assay technology were availble to format a high-throughput assay for the identification of Raf kinase inhibitors. A similar approach was also used by McDonald et al. at Glaxo-Welcome Inc., who identified Raf kinase inhibitors using a Raf/MEK/ ERK SPA HTS assay ${ }^{32}$."

The authors also wish to clarify that the primary objective of the randomized discontinuation trial (RDT) discussed on page 839 and 840 of the article was to test for disease-stabilizing activity of sorafenib in metastatic colorectal cancer (CRC), with secondary endpoints for activity in other solid tumour types using a design by Ratain. Analysis of the RDT data indicated no disease-stabilizing activity in metastatic CRC patients, but confirmed the early clinical signals observed in metastatic renal cell carcinoma (RCC) patients, and led to the study being refocused towards patients with metastatic RCC. 